
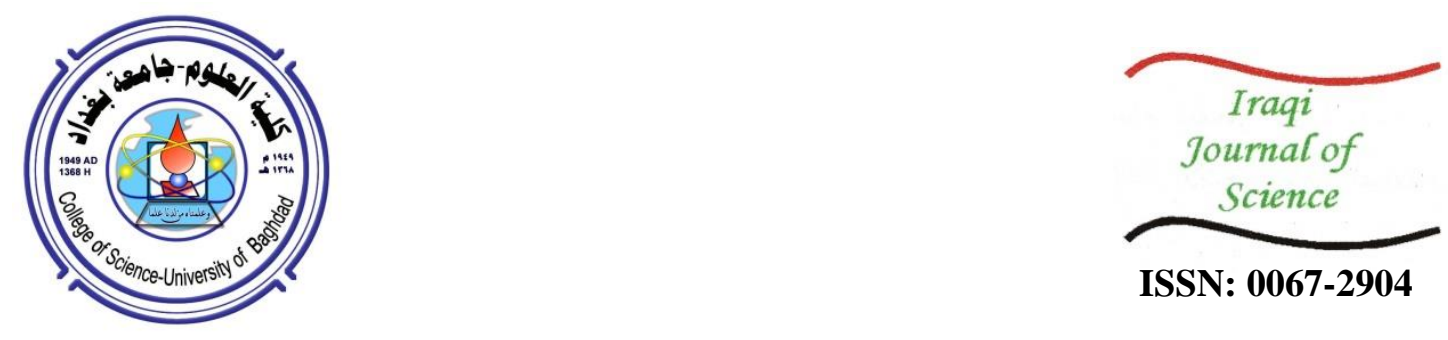

\title{
On the Construction of d-Koszul algebras
}

\author{
Ruaa Yousuf Jawad \\ Preparation of Trained Technicians Institute, Middle Technical University,Baghdad, Iraq
}

Received: 6/1/ 2020

Accepted: 15/3/2020

\begin{abstract}
An algebra $\boldsymbol{B}$ has been constructed from a (D, A)-stacked algebra $\boldsymbol{A}$, under the conditions that $D=d A, \mathrm{~A} \geq 1$ and $d \geq 2$. It is shown that when the construction of algebra $\boldsymbol{B}$ is built from a (D, A)-stacked monomial algebra $\boldsymbol{A}$ then $\boldsymbol{B}$ is a d-Koszul monomial algebra.
\end{abstract}

Keywords: Koszul Algebra, d-Koszul monomial algebra, and $(D, A)$-stacked monomial algebra.

$$
\begin{aligned}
& \text { حول بناء جبر الدي كاوزل } \\
& \text { رؤى يوسف جواد } \\
& \text { معهداعدادالددربين التقنين,الجامعه التقنيه الوسطى, بغداد, العراق. }
\end{aligned}
$$

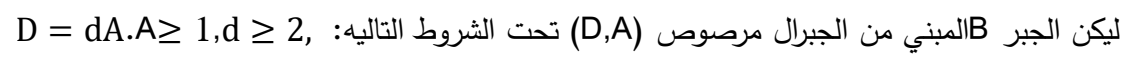

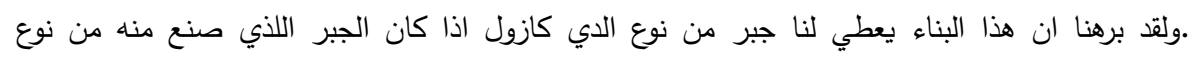

$$
\begin{aligned}
& \text { جبرأحادي الحدودالمرصوص. }
\end{aligned}
$$

\section{1-Introduction}

In this paper, we take a $(D, A)$-stacked algebra $\boldsymbol{A}=K \Gamma / \mathrm{I}$, where $\mathrm{D}=\mathrm{dA}, \mathrm{A} \geq 1$ and $d \geq 2$. We use that to construct an algebra $B$. The aim of this paper is to investigate the question whether each $\mathrm{d}$ Koszul algebra has been derived from a (D, A)-stacked algebra where $D=d A$ by using our construction. Leader [1] gives a construction of an algebra $\widetilde{\boldsymbol{\Lambda}}$ from a $d$-Koszul algebra $\boldsymbol{\Lambda}$ and she shows that if $\boldsymbol{\Lambda}$ is a $d$-Koszul algebra then $\widetilde{\Lambda}$ is a $(D, A)$-stacked algebra, where $D=d A$. Moreover, Jawad and Snashall [2] generalized this construction where they started with a finite dimensional algebra $\boldsymbol{\Lambda}=K Q / I$, for $A \geq 1$ and used it to construct a stretched algebra $\widetilde{\boldsymbol{\Lambda}}$. In this paper, the opposite of the above statement is investigated.

Furthermore, we show how the algebra $\boldsymbol{B}$ is constructed from a $(D, A)$-stacked algebra $\boldsymbol{A}$, and we prove that if $\boldsymbol{A}$ is an $(D, A)$-stacked monomial algebra, then $\boldsymbol{B}$ is $d$-Koszul where $D=d A$.

The paper begins with a background, while in section 3 we provide our construction where we begin with a $(D, A)$-stacked algebra $\boldsymbol{A}=K \Gamma / I$, we consider $D=d A, \mathrm{~A} \geq 1$ and $d \geq 2$, and we use the quiver $\Gamma$ and ideal $I$ to construct an algebra $B=K Q / \tilde{I}$.

Followed by detailed example of an algebra $\boldsymbol{A}=K \Gamma / I$, we construct the algebra $B$ from an algebra $\boldsymbol{A}$. This algebra will be shown to be a $d$-Koszul algebra.

The main idea of section 3 is Theorem 3.3, in which we prove that the finite dimensional algebra $\boldsymbol{B}$ is a d-Koszul monomial algebra.

Throughout this paper, we use a number of notations. We let $K$ be a field and let $\boldsymbol{A}=K \Gamma / \mathrm{I}$ be a finite dimensional (D, A)-stacked algebra, where $\Gamma$ is a finite quiver and $\mathrm{I}$ is an admissible ideal [3]. 
We denote the set of vertices of $\Gamma$ by $\Gamma_{0}$ and the set of arrows of $\Gamma$ by $\Gamma_{1}$, while we let $\operatorname{rad} A$ denotes the Jacobson radical of $\boldsymbol{A}$. An arrow $\alpha$ begins with the vertex $o(\alpha)$ and ends with the vertex $t(\alpha)$; the way to write the arrow in the path is from left side to right side. A path is a sequence $\alpha_{1} \alpha_{2} \cdots \alpha_{n}$ of arrows $\alpha_{1}, \alpha_{2}, \ldots, \alpha_{n}$ in $Q_{1}$, and the length of path $\mathrm{p}=\alpha_{1} \alpha_{2} \cdots \alpha_{n}$ is $\mathrm{n}$, which is denoted by $l(p)$. If the generating set of I is from a set of paths in $K \Gamma$, then we say that $\boldsymbol{A}=K \Gamma / \mathrm{I}$ is a monomial algebra [4].

Section 2: Background

We recall briefly the definition of Koszul algebra and we refer the reader to an earlier publication [5]. A graded algebra $\Lambda=\Lambda_{0} \oplus \Lambda_{1} \oplus \ldots$ is a Koszul algebra if $\Lambda_{0}=\Lambda / \mathrm{rad} \Lambda$ has a linear resolution; in other words, every projective module $P^{n}$ in the minimal graded projective resolution $\left(P^{n}, d^{n}\right)$ of $\boldsymbol{\Lambda}_{\mathbf{0}}$ can be generated in degree $n$. It was shown that if $\boldsymbol{\Lambda}=K Q / I$ is Koszul, then I is quadratic [5]. In another study [6], Berger introduced $d$-Koszul algebras. He was interested in this class of algebras to study Artin-Schelter regular algebras.

Definition 2.1 [6]. Let $\boldsymbol{\Lambda}=\Lambda_{\mathbf{0}} \oplus \boldsymbol{\Lambda}_{\mathbf{1}} \oplus \ldots$ be a graded K-algebra generated in degrees 0 and 1 . Assume that $\boldsymbol{\Lambda}_{\mathbf{0}}=\boldsymbol{\Lambda} / \mathrm{rad} \boldsymbol{\Lambda}$ is a finitely generated semisimple $K$-algebra, $\boldsymbol{\Lambda}_{\mathbf{1}}$ is a finitely generated $K$ module and that $\left(P^{n}, d^{n}\right)$ is a minimal graded

$\boldsymbol{A}$ - module projective resolution of $\boldsymbol{A} / \mathrm{rad} \boldsymbol{A}$. Let $d \geq 2$. We call $\boldsymbol{\Lambda}$ a d-Koszul algebra if, for all $n \geq 0, P^{n}$ is generated in single degree, $\delta(\mathrm{n})$, and

$$
\delta(\mathrm{n})= \begin{cases}\frac{n}{2} d & \text { if n even } \\ \frac{n-1}{2} d+1 & \text { if n odd }\end{cases}
$$

It can be seen that each Koszul algebra is a 2-Koszul algebra.

Green and Snashall [7] introduced the $(D, A)$-stacked monomial algebra. The case of non monomial (D, A)-stacked algebras was extended by Leader and Snashall [8].

Definition 2.2 [8, Definition 1.1]. Let $\boldsymbol{\Lambda}=K Q / I$ be a finite dimensional algebra.

Then $\boldsymbol{\Lambda}$ is a (D, A)-stacked algebra if there are natural numbers $D \geq 2, A \geq 1$ such that, for all $0 \leq n \leq$ gldim $\boldsymbol{\Lambda}$, the projective module $P^{n}$ in a minimal projective resolution of $\boldsymbol{\Lambda} / \mathrm{rad} \boldsymbol{\Lambda}$ is generated in degree $\delta(n)$, where

$$
\delta(\mathrm{n})= \begin{cases}0 & \text { if } n=0 \\ 1 & \text { if } n=1 \\ \frac{n}{2} D & \text { if n even } \\ \frac{n-1}{2} D+A & \text { if } n \text { odd }\end{cases}
$$

It can be seen that, if $A=1$, then the finite dimensional $(D, A)$-stacked algebras are $D$-Koszul algebras, as defined by Berger.

Green and Marcos [9] introduced the $\delta$-resolution determined algebras. Let $\boldsymbol{\Lambda}=K Q / I$ be a finite dimensional algebra, then $\Lambda$ is $\delta$-resolution determined, if there is a map $\delta: N \rightarrow N$ such that, for all $n>0$ with $n \leq \operatorname{gldim} \Lambda$, the projective module $P^{n}$ in a minimal projective resolution $\boldsymbol{\Lambda} / \mathrm{rad} \boldsymbol{\Lambda}$ can be generated in one degree $\delta(n)$.

Section 3: Constructing d-Koszul algebras

Now we take a (D, A)-stacked algebra $\boldsymbol{A}=K \Gamma / \mathrm{I}$ and we consider $D=d A$ and $\mathrm{d} \geq 2$,

where the set $\tilde{\rho}$ is a minimal generating set of homogeneous uniform relations of $I$ and each element of $\tilde{\rho}$ has a length D. The elements of $\tilde{\rho}$ are labelled as $\widetilde{\rho}_{1}, \ldots, \tilde{\rho}_{m}$. We are now ready to describe our construction using the quiver $\Gamma$ and ideal $\mathrm{I}$, and we show that the algebra

$\boldsymbol{B}$ is related to the algebra $\boldsymbol{A}$.

Definition 3.1:

1- We keep the notation above, and let $x \in K \Gamma$ where $x=\sum_{k} c_{k} \alpha_{k, 1} \cdots \alpha_{k, D}$ is a linear combination of paths of length $D$ with $D=d A, \quad 0 \neq c_{k} \in K$ and the $\alpha_{k, j} \in \Gamma_{1}$. We define the paths $\alpha_{k, r A+1} \cdots \alpha_{k,(r+1) A}$ to be the A-subpaths of $\mathrm{x}$ for some $\mathrm{k}$, and $0 \leq r \leq d-1$. 
2- We fix a minimal generating set $\tilde{\rho}$ for I. We define the $A$-subpaths of $\boldsymbol{A}$ to be the set of A-subpaths of $x$, for all $x \in \tilde{\rho}$, denoted as $S_{A}$, considering that a set $S_{A}$ is a set with no repeats. We define now a quiver $Q$ and an ideal $\hat{I}$ of $K Q$ and we let $B=K Q / \hat{I}$.

Definition 3.2: Let $\boldsymbol{A}=K \Gamma / I$ be a $(\mathrm{D}, \mathrm{A})$-stacked algebra and consider that $\mathrm{D}=\mathrm{dA}$

for some $\mathrm{d} \geq 2$, where the set $\tilde{\rho}=\left\{\tilde{\rho}_{1}, \ldots, \tilde{\rho}_{m}\right\}$ is a minimal generating set of uniform relations of a length $D$ of $I$. So for each $\mathrm{i}=\{1, \ldots, \mathrm{m}\}$, we write $\tilde{\rho}=\sum_{k} c_{k} \alpha_{i, k, 1} \cdots \alpha_{i, k, D}$ where $0 \neq c_{k} \in K$ and $\alpha_{i, k, j} \in \Gamma_{1}$, for all $1 \leq \mathrm{j} \leq \mathrm{D}$. Then

1- We define the vertex set of $Q$ to be the set $\left\{o\left(\tilde{\rho}_{i}\right), t(y)\right.$ for all $y \in S_{A}$ and all $i=\{1, \ldots, m\}$. We remark that $t\left(\alpha_{i, k, d A}\right)=t\left(\alpha_{i, k, D}\right)=t\left(\tilde{\rho}_{i}\right)$. In this set we do not have any repeated vertices, so if $t\left(\alpha_{i, k, r A}\right)=t\left(\alpha_{j, i, S A}\right)$ as vertices of $\Gamma$ or some $\mathrm{i}, \mathrm{j}, \mathrm{k}, \mathrm{l}, \mathrm{r}, \mathrm{s}$, then we classify $t\left(\alpha_{i, k, r A}\right)$ and $t\left(\alpha_{i, l, S A}\right)$ as the same vertex in $Q$.

2- The arrows of $Q$ are constructed in the following way. Each $y \in S_{A}$ corresponds to an arrow $\beta_{y}$ in $Q$. The diagram below illustrates this process.

Consider the path $\alpha_{i, k, 1} \cdots \alpha_{i, k, D}$. Then $e_{0}=o\left(\tilde{\rho}_{i}\right), e_{1}=t\left(\alpha_{i, k, A}\right), \ldots, e_{d}=t\left(\alpha_{i, k, d A}\right)$ are vertices in $Q$ and $\beta_{1}, \ldots, \beta_{d}$ are arrows in $Q$ corresponding to the A-subpaths

$$
\alpha_{i, k, 1} \ldots \alpha_{i, k, A}, \ldots, \alpha_{i, k,(d-1) A+1} \cdots \alpha_{i, k, d A}
$$

respectively. Then the $\alpha_{i, k, 1} \cdots \alpha_{i, k, D}$ may be considered as the path of the length $D$ in $K \Gamma$

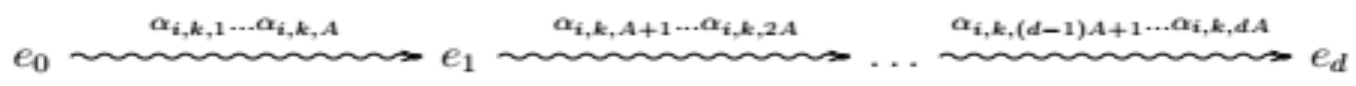

which corresponds to the path $\beta_{1} \ldots \beta_{d}$ of the length $d$ in $K Q$.

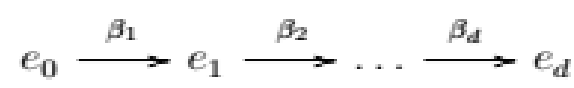

3- The ideal $\hat{I}$ of $K Q$ can be constructed as follows. For each $i=\{1, \ldots, m\}$, we define $\rho_{i}=$ $\sum_{k} c_{k} \beta_{k 1} \cdots \beta_{k d}$ in $K Q$ where $\beta_{j}$ is the arrow in $K Q$ which corresponds to a path $\alpha_{j, k, r A+1} \ldots \alpha_{j, k(r+1) A}$ for all $j=1, \ldots, d$ and $r=0, \ldots, d-1$. Now we define $\hat{I}$ to be the ideal of $K Q$ that is generated by the set $\rho=\left\{\rho_{1}, \ldots, \rho_{m}\right\}$. We remark that $\rho$ is certainly a minimal generating set for $\hat{I}$, since $\tilde{\rho}$ is a minimal generating set for $\hat{I}$. Let $\boldsymbol{B}=K Q / \hat{I}$. We illustrate this construction in the following example. Example 3.3: Let $\boldsymbol{A}=K \Gamma / I$ be the $(4,2)$-stacked algebra that is illustrated in the following quiver

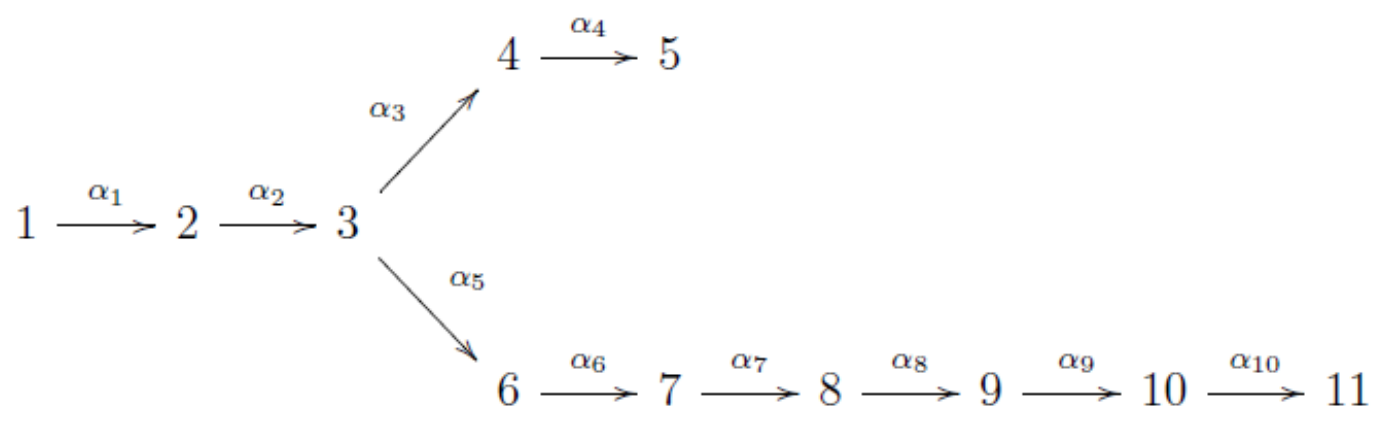

and with $I=\left\langle\alpha_{1} \alpha_{2} \alpha_{3} \alpha_{4}, \alpha_{1} \alpha_{2} \alpha_{5} \alpha_{6}, \alpha_{5} \alpha_{6} \alpha_{7} \alpha_{8}, \alpha_{7} \alpha_{8} \alpha_{9} \alpha_{10}\right\rangle$. We can see that the sets $g^{n}$ are given as follows: $g^{0}=\left\{e_{1} \ldots e_{11}\right\}, g^{2}=\left\{\alpha_{1} \alpha_{2} \alpha_{3} \alpha_{4}, \alpha_{1} \alpha_{2} \alpha_{5} \alpha_{6}, \alpha_{5} \alpha_{6}\right.$

$\left.\alpha_{7} \alpha_{8}, \alpha_{7} \alpha_{8} \alpha_{9} \alpha_{10}\right\}, g^{3}=\left\{\alpha_{1} \alpha_{2} \alpha_{5} \alpha_{6} \alpha_{7} \alpha_{8}, \alpha_{5} \alpha_{6} \alpha_{7} \alpha_{8} \alpha_{9} \alpha_{10}\right\}$, and $g^{4}=\left\{\alpha_{1} \alpha_{2}\right.$

$\left.\alpha_{5} \alpha_{6} \alpha_{7} \alpha_{8} \alpha_{9} \alpha_{10}\right\}$. We see that the length of the elements $g_{i}^{n} \in g^{n}$ is $\delta(\mathrm{n})$ where $D=4$ and $A=2$.

So every projective $P^{n}$ is generated in one degree $\delta(\mathrm{n})$. Hence, $\boldsymbol{A}$ is a $(D, A)$-stacked monomial algebra with $D=4, A=2$ and $d=2$. So $S_{A}=\left\{\alpha_{1} \alpha_{2}, \alpha_{3} \alpha_{4}, \alpha_{5} \alpha_{6}, \alpha_{7} \alpha_{8}, \alpha_{9} \alpha_{10}\right\}$. Then, by using the construction above, $Q$ has six vertices $o\left(\alpha_{1} \alpha_{2}\right), t\left(\alpha_{3} \alpha_{4}\right), t\left(\alpha_{5} \alpha_{6}\right), t\left(\alpha_{7} \alpha_{8}\right), t\left(\alpha_{9} \alpha_{10}\right)$ and five arrows $\left\{\beta_{1}, \beta_{2}, \beta_{3}, \beta_{4}, \beta_{5}, \beta_{6}\right\}$, where $\beta_{1}$ corresponds to $\alpha_{1} \alpha_{2}, \beta_{2}$ corresponds to $\alpha_{3} \alpha_{4}, \beta_{3}$ corresponds to $\alpha_{5} \alpha_{6}, \beta_{4}$ corresponds to $\alpha_{7} \alpha_{8}$, and $\beta_{5}$ corresponds to $\alpha_{9} \alpha_{10}$. Therefore, $B=K Q / \bar{I}$ is given by the quiver 


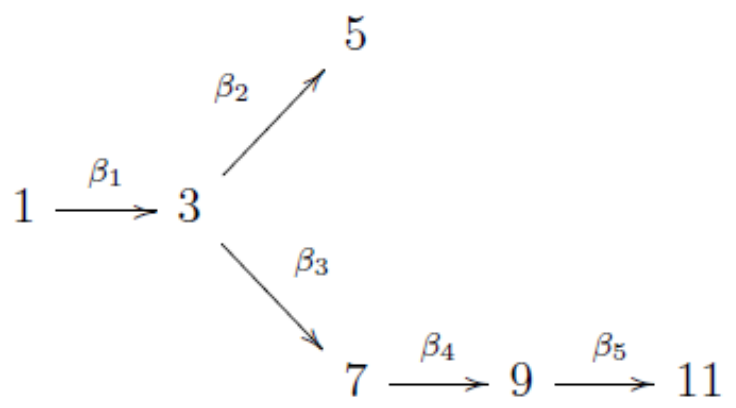

and $\hat{\mathrm{I}}=\left\langle\beta_{1} \beta_{2}, \beta_{1} \beta_{3}, \beta_{3} \beta_{4}, \beta_{4} \beta_{5}\right\rangle$. So $\boldsymbol{B}$ is a Koszul algebra (see [10]). More examples can be found in a previous article [11].

Section 4: $d$-Koszul monomial algebra

In this section, the algebra $\boldsymbol{B}$, which has been constructed from a $(D, A)$-stacked monomial algebra, will be shown to be $d$-Koszul. Before that, the idea of overlaps has been used to prove the main theorem in this section. Green and Zacharia [10] have used the concept of overlaps to describe a basis of the Ext algebra of a monomial algebra.

We consider that $\boldsymbol{A}=K Q / I$ is a monomial algebra.

Definition 4.1 [7]. Let $q$ and $p$ be paths. Then we say that a path $q$ overlaps a path $p$ with an overlap , if there are paths $u$ and $v$ such that $p u=v q$ and $1 \leq l(u) \leq l(q)$. The definition can be shown in the following diagram:

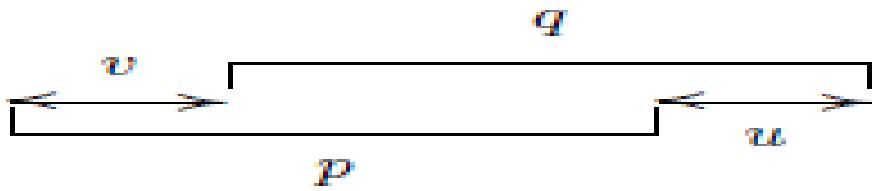

Thus, the two paths allow $l(v)$ to be zero.

2- A path $q$ properly overlaps a path $p$ with an overlap $p u$ if $q$ overlaps $\mathrm{p}$ and $l(v) \leq 1$.

3- A path $p$ has no overlaps with a path $q$ if $p$ does not properly overlap $q$ and q does not properly overlap $p$.

Definition 4.2 [12]. A path $p$ is a prefix of a path $q$ if there is some path $p$ such that $q=p p^{\prime}$.

The minimal projective resolution $\left(P^{n}, d^{n}\right)$ of $A / \mathrm{radA}$ has been constructed by Green, Happel and Zacharia [4], by using overlaps. Moreover, it has been further described by other authors [12,10]. The same previously demonstrated notation [12] has been used in this paper. The sets $\mathcal{R}^{n}$ can be defined as follows:

$\mathcal{R}^{0}=$ the vertices set of $Q$,

$\mathcal{R}^{1}=$ the arrows set of $Q$

$\mathcal{R}^{2}=$ the minimal set of monomials in the generating set of $I$.

For all $n \geq 3, R^{2} \in \mathcal{R}^{2}$ maximally overlaps $R^{n-1} \in \mathcal{R}^{n-1}$ with overlap

$R^{n}=R^{n-1} u$ for some $u \in K Q$, if it satisfies the following conditions:

$1-R^{n-1}=R^{n-2} p$, for some path $p$ in $K Q$;

2- $R^{2}$ overlaps $p$ with overlap $p u$;

3- There is no element in $\mathcal{R}^{2}$ which overlaps $p$, with the overlap being a proper prefix of $p u$.

The set $\mathcal{R}^{n}$ is the set of all overlaps $R^{n}$. We illustrate $R^{n}$ with the following diagram:

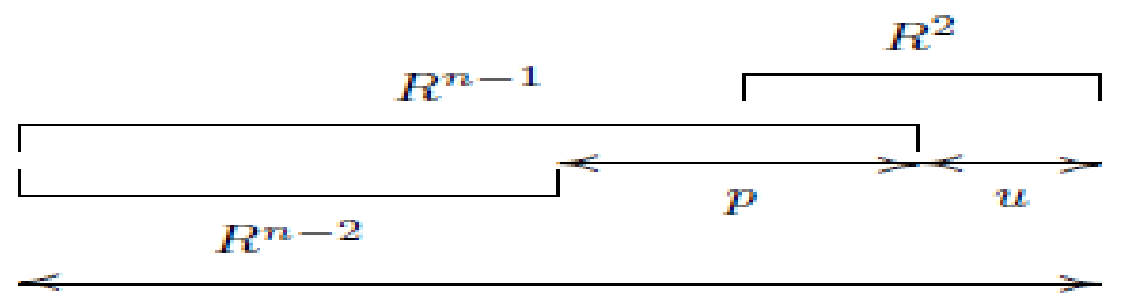


Now we define the minimal projective resolution $\left(P^{n}, d^{n}\right)$ of $\Lambda / \mathrm{rad} \Lambda$ using the construction of Green, Happel and Zacharia [4]. For all $n \geq 0$, let $P^{n}=\bigoplus_{R^{n} \in \mathcal{R}^{n}} t\left(R^{n}\right) \Lambda$. Define, for $n \geq 1$ and $R^{n} \in \mathcal{R}^{n}$, the map $d^{n}: P^{n} \rightarrow P^{n-1}$ via $t\left(R^{n}\right) \rightarrow(0, \ldots, 0, p, 0, \ldots)$, where $R^{n}=R^{n-1} p$ and $p$ occurs in the component of $P^{n-1}$ corresponding to $R^{n-1}$.

Now we prove the main theorem in this section.

Theorem 4.3: Let $\boldsymbol{A}=K \Gamma / I$ be a (D, A)-stacked monomial algebra with gldim $\boldsymbol{A} \geq 4$, so $D=d A$, for some $d \geq 2$. Let $\boldsymbol{B}$ be the algebra constructed from $\boldsymbol{A}$ using Definition 3.2. Then $\boldsymbol{B}$ is a $d$-Koszul monomial algebra.

Proof: Assume that the algebra $\boldsymbol{B}=K Q / \mathrm{I}$ is constructed from $\boldsymbol{A}=K \Gamma / I$. Then Î is monomial. We set

$\tilde{\mathcal{R}}^{0}=$ the vertices set of $\Gamma$,

$\tilde{\mathcal{R}}^{1}=$ the arrows set of $\Gamma$,

$\widetilde{\mathcal{R}}^{2}=$ the minimal set of monomials in the generating set of $I$, (denoted $\tilde{\rho}$ in Definition 3.2) and

$\mathcal{R}^{0}=$ the vertices set of $\mathrm{Q}$,

$\mathcal{R}^{1}=$ the arrows set of $\mathrm{Q}$,

$\mathcal{R}^{2}=$ the minimal set of monomials in the generating set of $\hat{\mathrm{I}}$, (denoted as $\rho$ in Definition 3.2).

It can be seen that $l\left(R^{0}\right)=0, l\left(R^{1}\right)=1$, and $l\left(R^{2}\right)=d$, for all $R^{0} \in \mathcal{R}^{0}, R^{1} \in \mathcal{R}^{1}$,

and $R^{2} \in \mathcal{R}^{2}$. We denote by $\tilde{\mathcal{R}}^{n}$ (respectively $\mathcal{R}^{n}$ ) the set of overlaps in $\boldsymbol{A}$ (respectively $\boldsymbol{B}$ ), for all $n \geq 3$. Since $\boldsymbol{A}$ is a $(D, A)$-stacked monomial algebra, then the $n$th projective module in a minimal resolution of $\boldsymbol{A} / \mathrm{rad} \boldsymbol{A}$ is $\tilde{P}^{n}=\bigoplus_{R^{n} \in \tilde{R}^{n}} t\left(\tilde{R}^{n}\right) A$, and we can see that it is generated in single degree $\delta(n)$ (see Definition 1.3), where $n \geq 0$. We now consider $\mathcal{R}^{3}$. An element $R^{3} \in \mathcal{R}^{3}$ is constructed from $R_{1}^{2}$ which maximally overlaps $R_{2}^{2}$ of the form $R^{3}=R_{2}^{2} y$ as follows:

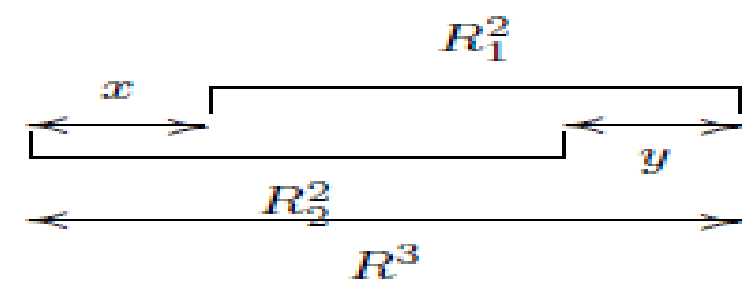

By Definition 2.2, $R_{1}^{2}$ (respectively $R_{2}^{2}$ ) corresponds to $\tilde{R}_{1}^{2}$ (respectively $\tilde{R}_{2}^{2}$ ) in the minimal generating set $\widetilde{\mathcal{R}}^{2}$ for $I$. This element gives an overlap of $\widetilde{R}_{1}^{2}$ with $\widetilde{R}_{2}^{2}$ :

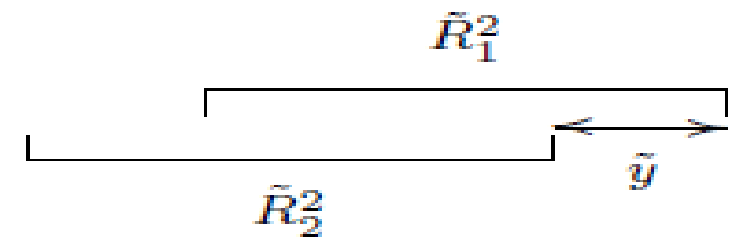

We want to show that $\tilde{R}_{2}^{2} \tilde{y}$ is in $\tilde{R}^{3}$. If $\tilde{R}_{1}^{2}$ does not maximally overlap $\tilde{R}_{2}^{2}$, then we have $\tilde{R}_{3}^{2} \in \tilde{R}^{2}$ which maximally overlaps $\tilde{R}_{2}^{2}$ :

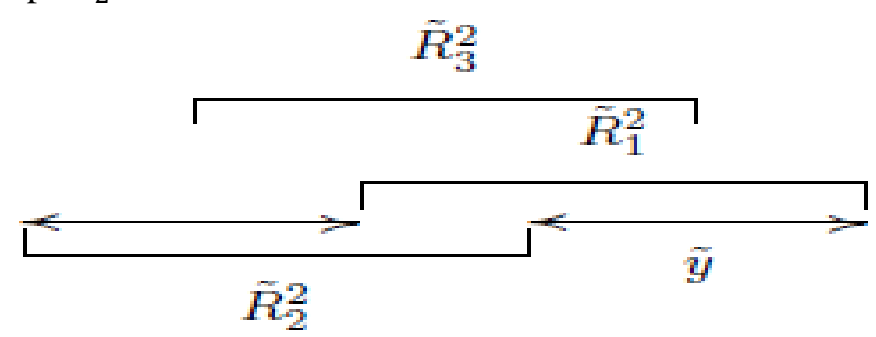

But $\widetilde{R}_{3}^{2}$ corresponds to some $R_{3}^{2} \in \mathcal{R}^{3}$ and so $R_{3}^{2}$ overlaps $R_{2}^{2}$ : 


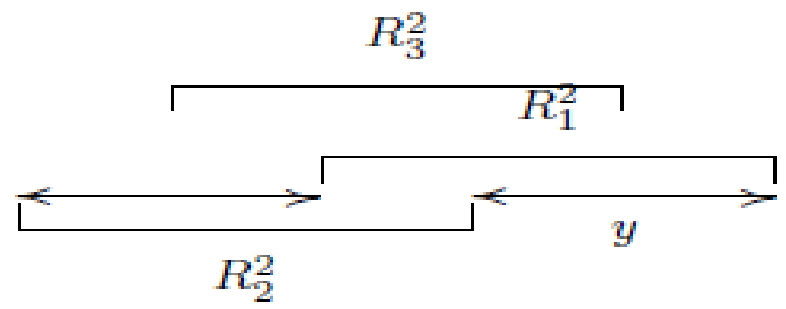

This is a contradiction, as $R_{1}^{2}$ maximally overlaps $R_{2}^{2}$. Therefore, $\tilde{R}_{1}^{2}$ does maximally overlap $\tilde{R}_{2}^{2}$ and so $\tilde{R}_{2}^{2} \tilde{y} \in \tilde{\mathcal{R}}^{3}$. Now we write $\tilde{R}_{2}^{2} \tilde{y}=\tilde{R}^{3} \in \tilde{\mathcal{R}}^{3}$. Since $l\left(\tilde{R}^{3}\right)=D+A$ and $l\left(\tilde{R}_{2}^{2}\right)=D$, then $l(\tilde{y})=A$. However, $\tilde{y}$ is a suffix of $\tilde{R}_{1}^{2}$ and so $\tilde{y} \in S_{A}$. So $\tilde{y}$ corresponds to an arrow in B. Hence, $l(y)=1$ and $l\left(R^{3}\right)=d+1$. The same argument has been used for the elements of $\mathcal{R}^{4}$. An element $R^{4} \in \mathcal{R}^{4}$ is constructed from a sequence of overlaps as follows:

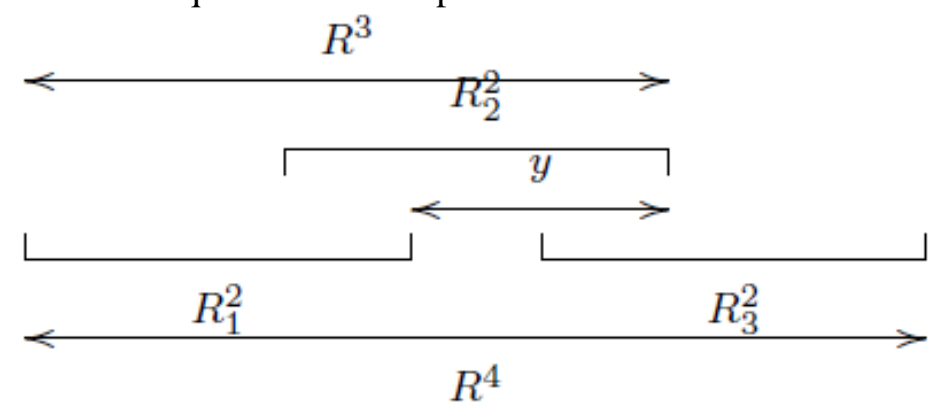

From Definition 3.2, $R_{1}^{2}$ (respectively $R_{2}^{2}, R_{3}^{2}$ ) corresponds to

$\tilde{R}_{1}^{2}$ (respectively $\tilde{R}_{2}^{2}, \tilde{R}_{3}^{2}$ ) in the minimal generating set $\tilde{\mathcal{R}}^{2}$ for $I$. Using the above argument for $\tilde{R}^{3}$, we get:

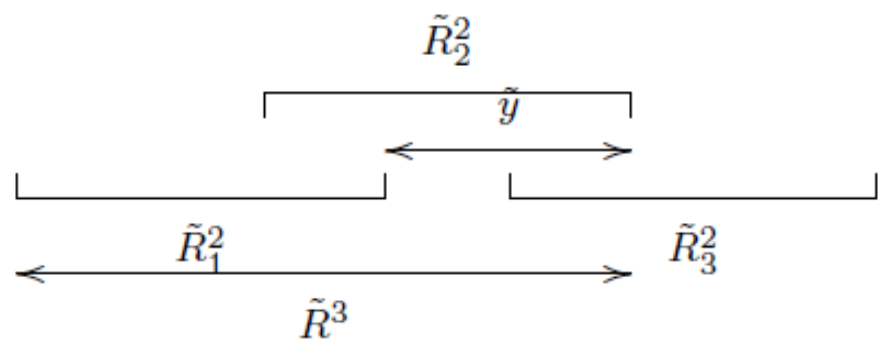

and $l(\tilde{y})=A$. From our construction of $R^{4}$, we get $l(y)=1$. However, $R_{3}^{2}$ overlaps $y$ and so $y$ must be a prefix of $R_{3}^{2}$. Thus $t\left(R_{1}^{2}\right)=o\left(R_{3}^{2}\right)$ and we have

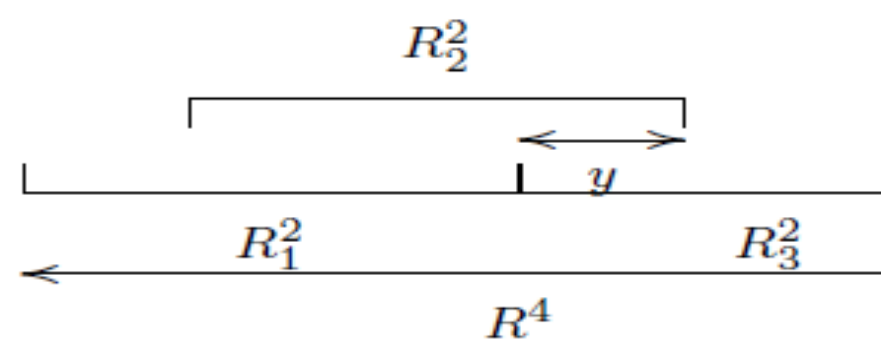

Then, $R^{4}=R_{1}^{2} R_{3}^{2}$ and $l\left(R^{4}\right)=2 d$. By continuing in the same manner, by the induction for all $n \geq 0$ and all $R^{n} \in \mathcal{R}^{n}$, we have

Thus, $\boldsymbol{B}$ is a $d$-Koszul.

$$
l\left(R^{n}\right)= \begin{cases}\frac{n}{2} d & \text { if n even } \\ \frac{n-1}{2} d+1 & \text { if } n \text { odd }\end{cases}
$$




\section{Acknowledgement}

I would like to deliver a special thank you to my supervisor, Professor Nicole Snashall, for her guidance.

\section{References}

1. Leader, J. 2014. Finite generation of Ext and (D, A)-stacked algebras, PhD thesis, University of Leicester.

2. Jawad, R. And Snashall, N. 2010. Hochschild cohomology, finiteness conditions and a generalisation of d-Koszul algebras, preprint.

3. Assem, I. And Simson, D. 2006. A. Skowroński, Elements of the Representation Theory of Associative Algebras: 1, LMS Student Texts 65, CUP.

4. Green, E.L., Happel, D. and Zacharia, D. 1985. Projective resolutions over Artin algebras with zero relations, Illinois J. Math. 29(1): 180-190.

5. Green, E.L. and Martínez-Villa, R. 1994. Koszul and Yoneda algebras, Representation theory of algebras (Cocoyoc,1994), 247-297, CMS Conf. Proc. 18, Amer. Math.

6. Berger, R. 2001. Koszulity of nonquadratic algebras, J. Algebra, 239: 705-734. Soc., Providence, RI.

7. Green, E.L. and Snashall, N. 2006. Finite generation of Ext for a generalization of D-Koszul algebras, J. Algebra. 295: 458-472.

8. Leader, J. and Snashall, N. 2017. The Ext algebra and a new generalisation of D-Koszul algebras, Quart. J. Math. 68: 433-458.

9. Green, E.L. and Marcos,E.N. 2005. d-Koszul Algebras, Comm. Algebra, 33: 1753-1764.

10. Green, E.L. and Zacharia, D. 1994. The Cohomology Ring of a Monomial Algebra, Manuscripta Math. 85: 11-23.

11. Jawad, R. 2019. Cohomology and fniteness conditions for generalisations of Koszul algebras, $\mathrm{PhD}$ thesis, University of Leicester.

12. Green,E.L. and Snashall, N. 2006. The Hochschild cohomology ring modulo nilpotence of a stacked monomial algebra, Colloq. Math. 105: 233-258. 